\title{
New Investigator Award: announcing our finalists!
}

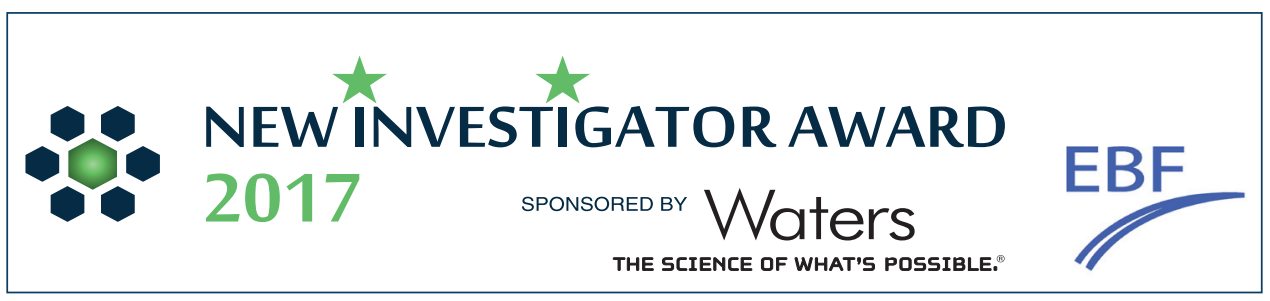

Over the past 7 years, Bioanalysis and Bioanalysis Zone have been proud to host the New Investigator Award, to pinpoint and promote promising early-career researchers in our community. The time has now come for you to select your winner for the New Investigator Award 2017.

We are delighted to present our judges' selection of finalists (in alphabetical order):

- Swarnapali De Silva Indrasekara (Duke University, NC, USA)

- Matthew Ryen Lockett (University of North Carolina at Chapel Hill, NC, USA)

- Michael Witting (Helmholtz Zentrum Muenchen/Habilitation at the Technical

University, Munich, Germany)

- Yu Shrike Zhang (Harvard Medical School, MA, USA)

You can view the finalists' mini profiles below. To view Swarnapali, Matthew, Michael and Yu's full profiles and short interviews, please visit the Bioanalysis Zone website. The 2017 Award is being held in the association with Waters Corporation (MA, USA), with the winner receiving a US $\$ 1000$ prize and a year's subscription to Bioanalysis. The winner will be announced and presented with the award at the European Bioanalysis Forum open meeting taking place in Barcelona (Spain) in November this year, where they will also present their work.

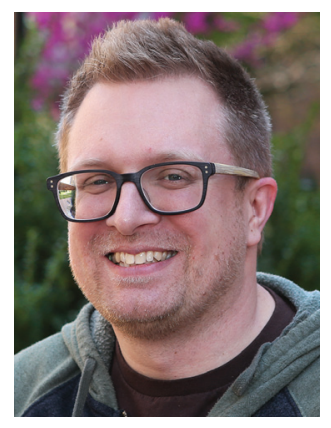

\section{Matthew Ryen Lockett mlockett@unc.edu}

Supporting comments:

Matthew has a thriving research group focused on several aspects of measurement science. His lab is developing $3 \mathrm{D}$ tissue- and tumorlike models to study the role of oxygen, and oxygen-regulated pathways, in directing cellular movement (e.g., as a chemoattractant) and promoting drug resistance. The work combines analytical sensors for measuring the extracellular and intracellular environment. The cultures are prepared using paper-based scaffolds, and through a series of creative fabrication steps can generate up to $963 \mathrm{D}$ cultures in parallel, making it an interesting and readily accessible platform for screening cellular responses to chemotherapies. His work will have broad implications in both understanding the biochemical role of oxygen in cancer progression, and in generating technologies for the assessment and treatment of potentially invasive tumors.

\section{newlands}

press part of 
Nominated by:

Jeff Johnson, Department of Chemistry, University of North Carolina at Chapel Hill, NC, USA; Tel.: +1 919

962 4358; jsj25@email.unc.edu

Q Describe the main highlights of your bioanalytical work

My career can be divided into vignettes, each which has shaped my current research trajectory. As a graduate student, the carbon-on-metal substrates I developed could support the label-free imaging of thousands of unique oligonucleotide features in a single experiment. These arrays showed me the power of screening multiple reactions in parallel, quantifying interactions of surfacebound oligonucleotides with mixtures containing DNA, RNA and proteins. I also developed a suite of chemistries to immobilize biomolecules on the carbon-on-metal substrates (16 papers, 11 first-author).

As a postdoc, I utilized different biophysical techniques to determine the role of water in defining the binding affinity of protein-ligand interactions. This work combined protein expression and characterization with measurement techniques such as ultracentrifugation, calorimetry and fluorescence-based binding assays (ten papers, five first-author).

My independent lab builds upon another aspect of my postdoctoral experience, constructing 3D tissue models. We are currently developing a 3D culture platform to quantify how oxygen gradients direct cellular invasion and promote drug resistance in tumor-like structures. We use fluorescence-based sensors to measure oxygen and $\mathrm{pH}$ gradients in these cultures, and have developed a number of techniques to quantify cellular movement (eight papers, five independent lab).

Q How has your work impacted your laboratory, the bioanalytical field \& beyond?

Each of my research experiences has shaped the work that my laboratory is currently engaged in. I have two very active subgroups, focused on: the fabrication, chemical modification and characterization of carbonbased electrodes for biosensing and catalysis applications; and the generation of paper-based 3D cultures to study the role of oxygen gradients in directing cellular invasion, promoting drug resistance and regulating protein expression.

Our work lies at the interface of many disciplines, but relies making accurate measurements. The tools we are generating will result in screening technologies capable of evaluating new pharmaceutical candidates and potential toxicants in tissue-like structures. These technologies will provide a more predictive means of evaluating cellular responses to compounds.

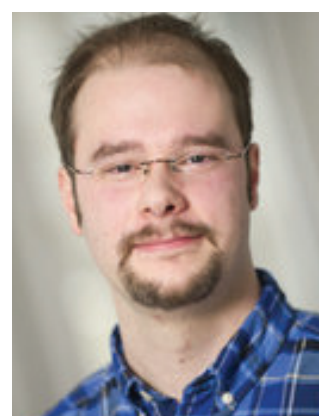

\section{Michael Witting michael.witting@helmholtz-muenchen.de Supporting comments:}

Hereby, I nominate M Witting for the Bioanalysis Zone New Investigator Award. During the last years, he developed new analysis methods for the metabolome of the model organism Caenorhabditis elegans. In future, these methods will enable a deeper insight into the metabolic regulations in this model organism in health and disease and will advance our understanding of the nematodes biology. His work published in 2015 in Analytical and Bioanalytical Chemistry aimed to develop a Direct-InfusionFourier-Transform-Mass Spectrometry workflow for the in-depth analysis of the $C$. elegans metabolome in short time. His method offers high sensitivity and allows the analysis from a few microliters of an extract corresponding to 20 worms $/ \mathrm{ml}$, which calculates back to only a few thousand cells. This lays the foundation of single-worm metabolomics, which will enable future large-scale screening efforts to be combined with metabolomics. The approach not only influences the way $C$. elegans metabolomics will be performed in future, moving from population-based formats to individual worms but also metabolomics in general. The combination of (micro-)metabolomics with C. elegans research is unique and deserves distinction with this award. Please do not hesitate to contact me for further details.

Nominated by:

Philippe Schmitt-Kopplin, Helmholtz Zentrum Muenchen, Ingolstaedter Landstrasse 1, 85764 Neuherberg, Germany; Tel.: +0049 893187 3246; schmitt-kopplin@helmholtz-muenchen.de

Q Describe the main highlights of your bioanalytical work

During my $\mathrm{PhD}$, I developed and applied extrac- tion and analysis methods for C. elegans samples and applied them to a C. elegans-Pseudomonas aeruginosa infection model. Several publications resulted from 
the obtained data (Wägele et al. 2013, Witting et al. 2015, Witting et al. 2014, Witting et al. 2017).

A special focus was the use of DI-FT-ICR-MS as fast metabotyping tool. Based on the obtained metabolic snapshots, I was not only able to separate worms infected with $P$. aeruginosa PA14 from control worms but also the attenuated $\triangle$ gacA mutants from the fully virulent wildtype and from Salmonella enterica infection and starvation as additional measures of stress. This method can now serve as a new tool in screening in infection biology using C. elegans as model host and metabolism as functional readout. The short analysis time of less than 10 min per sample enables a high throughput. Furthermore, for measurement the metabolite extracts have to be diluted to an equivalent of 20 worms $/ \mathrm{ml}$, which enables the use of lower biomass and is an additional important point for the integration in high-throughput studies.

Q Describe the most difficult challenge you have encountered in your scientific career \& how you overcame it

One particular challenge was to find an own topic for my future research. I wanted to further develop myself and apply the skills I have learned during my diploma and doctoral thesis to new bioanalytical problems. However, I also wanted to differentiate myself from my supervisors.

During my doctoral thesis, I was working for the first time with $C$. elegans and I realized that the combination of a genetic tractable model organism like the worm with metabolomics is a fruitful combination.

Even up to the present day not many laboratories combine these two. Therefore, I decided to focus on C. elegans metabolomics as my future main topic. In 2013, I was searching for new collaboration partners within this topic to strengthen my scientific profile and enable myself to learn more about the worm's biology.

My collaboration with Hugo Aguilaniu is among one of the most fruitful established links and I successfully acquired funding from the DAAD for scientific exchange with the Aguilaniu lab at the ENS in Lyon. During research visits at this lab, I learned all necessary techniques to set up my own $C$. elegans cultivation facilities and by this I gained independence.

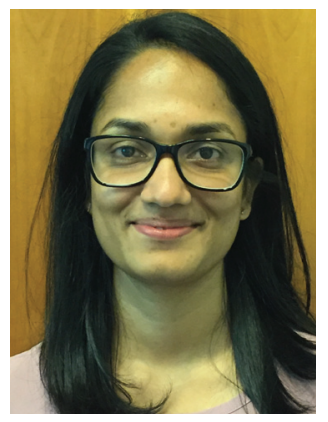

\section{Swarnapali De Silva Indrasekara a.pali.dsi@gmail.com \\ Supporting comments:}

Indrasekara is the best student I have supervised and among the top 1\% I have come across in my academic career. Pali's dissertation focused on the development of nanostructured platforms for SERS-based sensing and cell imaging and on the fundamental study of SERS enhancement in assembled nanostructures, and produced six manuscripts. In addition to her contributions within the group, Pali excelled at establishing and maintaining collaborations, the major of which has been her work with the Metropolitan Museum of Art of New York City. During her postdoc at Rice University, Pali shifted her focus from applied to fundamental science, to investigate nanoscale processes using single particle/molecule spectroscopic techniques and gain insight into the dynamic behavior of bio-nanointerfaces. As a postdoc at Duke University, Pali has been working with clinicians of Duke's Medical School to design and deliver effective therapeutic nanomaterials and to design low-cost nanobiosensors for point-of-detection applications. Pali's talent, perseverance, creativity and ingenuity are admirable. Her research is thorough and carefully carried out, and demonstrates her depth of understanding on both fundamental and applied concepts. Based on my observations of her, Pali promises to make outstanding contributions in the fields of bioanalytical chemistry and materials science.

Nominated by:

Laura Fabris, Rutgers University, Materials Science and Engineering, 607 Taylor Road, Piscataway, NJ, USA; Tel.: +1 848445 5606; lfabris@soe.rutgers.edu

\begin{abstract}
Q Describe the main highlights of your bioanalytical work

I implement my expertise in nanochemistry and spectroscopy as analytical tools to innovate new technologies to understand processes significant in molecular biology and medicine. Surface-enhanced Raman spectroscopy (SERS) is the main analyti-
\end{abstract}

cal tool of my interest, which is noninvasive and can be utilized even for single-molecule detection under optimized conditions. My research niche lies at the interface of fundamental science and materials engineering to realize improved analytical measures for SERS-based bioanalytical and biomedical applications. 
I developed a US patent-granted chip technology for direct, light-mediated detection of bio/chemical molecules in femtomolar regime, and multiplexed detection in nanomolar regime.

Detection of cancers at very early stages is important for effective therapies. I developed nanoprobes as SERS tags that can differentiate healthy cells from disease cells based on the cancer biomarker expression levels on cell membranes using SERS signal as the feedback.

I contributed to a study that uses super-resolution fluorescence microscopy as a qualitative and semiquantitative analytical technique to gain a mechanistic understanding of therapeutic nanoparticles-biomolecules interactions in situ and assess biosafety of nanomaterials. This study reveals the loss of structural integrity of a serum protein on a single nanoparticle, providing unique information that could not otherwise be obtained from other ensemble analytical techniques. This work highlights the strength of single-particle analytical tools in assessing biosafety.

\section{Q Describe your role in bioanalytical} communities/groups

I am involved in several interdisciplinary collaborative research efforts that integrate plasmonic nanomateri- als-based analytical techniques in medical diagnostics and imaging. I invest my expertise in analytical performance-oriented materials development, and Raman spectroscopy-based sensing technologies to unveil mechanistic understandings of the molecular biology of invasive cancers.

I am also involved in projects as a part of the global health imitative to device field-deployable diagnostic schemes for mosquito-borne infectious diseases. My role in this team as an analytical chemist is to fabricate the underlying chemistry of the biosensing assays to realize higher specificity and sensitivity for biomarkers in complex matrices such as blood.

I am also advising junior researchers from engineering background to facilitate their transition into applied science. In that regard, I guide them in controlling drug-retention time/release profile in nanocapsules by proper engineering of surface chemistries.

In addition, I have also been participating in science outreach events to increase the awareness of the young generation on the impact of light-based technologies in global health. I have introduced several high school students to the optics-based sensing and diagnostics technologies, and also involved as a spokesperson for the Duke-light technology event for kids, sharing the multifaceted nature of photonics-based technologies in medicine.

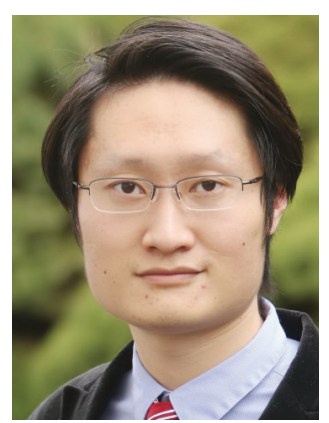

\section{Yu Shrike Zhang} yszhang@research.bwh.harvard.edu Supporting comments:

I am writing with greatest enthusiasm to nominate Zhang for the Award. Shrike's talents are clearly reflected in his more than 80 publications, more than 2300 citations, an h-index of 5 and more than 20 honors/awards. Among all the trainees I have worked with, I rank Shrike as the top one. Specifically, Shrike has developed the best organs-on-chips platform I have ever seen in the world with well-designed organoid microbioreactors, microfluidic circuitry and controlling systems. Most importantly, various in-line biosensors have also been innovated for integration to perform, for the first time, continual analyses of physical parameters and secreted biomarkers of organoids for screening drugs in a fully automated manner. This system aims to provide an innovative approach for personalized drug screening, potentially inducing a paradigm shift in the landscape of pharmaceutical industry to improve the human healthcare. This work was recently published in PNAS. He took an independent faculty position of Instructor at Brigham/HMS earlier in 2016, at the youngest age ( $<30$ by time of appointment), I have ever known for this level of position. I view Shrike as the brightest early-stage investigator among his peers and have very high expectations for his success in advancing the field of precision medicine.

Nominated by: Ali Khademhosseini, Brigham and Women's Hospital, Harvard Medical School, 65 Landsdowne Street, PRB 252, Cambridge, MA, USA; Tel.: +1 617768 8330; alik@bwh.harvard.edu

\section{Q Describe the main highlights of your} bioanalytical work

In my graduate research, I pioneered the applications of photoacoustic microscopy in noninvasive, volumetric characterization of tissue-biomaterial interac- tions both in vitro and in vivo. During my postdoctoral training, I developed a series of novel-on-chip biosensing platforms by integrating sophisticated microfluidic circuitry and valve/flow controllers to achieve, for the first time, fully automated, continual 
and multiplexed monitoring of organoid behaviors for drug-screening applications. These biosensing platforms include miniature imagers for characterizing the morphological alterations of the organoids, physical sensors for monitoring microenvironmental parameters (e.g., $\mathrm{pH}$, oxygen, temperature) and biochemical sensors for measuring organoid-secreted soluble biomarkers at trace concentrations. These microfluidic biosensors are critical in providing realtime information of the biological responses of the organoids toward pharmaceutical compounds over extended periods of time without human interference. I have also invented a transformative technology termed the expansion mini-microscopy, which elegantly combines physical expansion of biological specimens with mini-microscopy for high-resolution imaging at extremely low cost. I am now carrying these advanced bioanalytical techniques into the research of my recent independence as a junior faculty with a shift in focus toward cancer precision therapy, by producing patient cancer models equipped with high-capacity biosensors for personalized chemotherapeutic drug screening.

Q Describe the most difficult challenge you have encountered in your scientific career \& how you overcame it

The most difficult challenges throughout my career in bioanalysis happen to coincide with each other - that is, to establish an entirely new direction with limited prior experiences to learn from. At the early stage of my grad- uate research, I was not able to defend my hypothesis that uniform 3D scaffolds induce better cell distribution and angiogenesis than nonuniform counterparts, because cryosectioning often crushed the scaffolds instead of cutting them into thin pieces that could be observed under optical microscopy. Fortunately, I took an unusual way to solve this problem by switching to photoacoustic microscopy, necessitating no more sectioning. With these experiences, I felt comfortable to do it again in my postdoctoral training by building up a project from scratch and gearing it toward microfluidics-integrated biosensing, an exciting new direction for me and the laboratory then. After 3 years of efforts, I developed several multiplexed biosensing platforms capable of continual, noninvasive analysis of biophysical and biochemical parameters in organoid cultures. This most difficult challenge - setting up a research direction with minimal guidance - has well prepared me to transition into recent independence, where I am taking another new direction of applying these bioanalytical tools to cancer-precision medicine.

\section{Financial \& competing interests disclosure}

The authors have no relevant affiliations or financial involvement with any organization or entity with a financial interest in or financial conflict with the subject matter or materials discussed in the manuscript. This includes employment, consultancies, honoraria, stock ownership or options, expert testimony, grants or patents received or pending, or royalties.

No writing assistance was utilized in the production of this manuscript. 